“(C) 2015 IEEE. Personal use of this material is permitted. Permission from IEEE must be obtained for all other uses, in any current or future media, including reprinting/republishing this material for advertising or promotional purposes, creating new collective works, for resale or redistribution to servers or lists, or reuse of any copyrighted component of this work in other works." 


\title{
Optimal Energy Efficiency Link Adaptation in IEEE 802.15.6 IR-UWB Body Area Networks
}

\author{
Author 1, Author 2, Author 3, and Author 4
}

\begin{abstract}
We propose a novel link adaptation mechanism to maximize energy efficiency in IEEE 802.15.6 impulse radio ultra wideband (IR-UWB) wireless body area networks (WBANs). We consider noncoherent energy detection and autocorrelation receivers, suitable for low complexity implementations. The amount of captured energy is first modeled for the on-body WBAN channel. Using our energy capture model and Gaussian approximations for the decision statistic, the error performance of various physical layer modes of the IEEE 802.15.6 standard is derived assuming intra-symbol interference. We refer to the IEEE 802.15.6 specification as a use case. The proposed adaptation scheme can be applied to any other IR-UWB system with noncoherent receivers and is based on the estimated signal to noise ratio and the channel's energy capture index for which we propose unbiased estimators.
\end{abstract}

Index Terms-Wireless Body Area Networks, UWB, IEEE 802.15.6, Link Adaptation

\section{INTRODUCTION}

The mandatory physical layer (PHY) scheme in the recent IEEE 802.15.6 standard is IR-UWB, which consists of the default and high quality of service (QoS) PHY options for general and high priority medical applications, respectively [1]. Both PHY options allow for a set of different modulation and coding techniques to obtain various data rates. However, the question of how to coordinate the available data rates in an optimal manner, referred to as rate adaptation or link adaptation (LA), remains unanswered.

A link adaptation method based on the estimated frame error rate is proposed in [2] for multiband OFDM UWB systems. Also in [3] LA is used to improve system throughput in OFDM based UWB systems. In [4] and [5], SNR-based LA strategies are proposed to reduce packet error probability in narrowband IEEE 802.15.4 networks. Also in [6], interference mitigation in WBAN applications is performed by means of data rate adaptation. However, to the best of our knowledge none of the existing works address LA in IR-UWB systems. The benefit of this approach is sensitivity to variations in channel conditions which is crucial in WBAN applications. Due to the strict limitations in a typical WBAN, energy efficiency is a crucial issue and any approach that could optimize the overall energy efficiency of the system is appealing. For this reason the objective of the proposed LA scheme is to maximize energy efficiency. Nevertheless, the proposed framework can directly be applied to any other LA approach in noncoherent IR-UWB systems other than IEEE 802.15.6 with a different objective (e.g. Throughput).

Author 1 is with -

Author 2 is with -

Author 3 is with -

Author 4 is with -
The contributions of this letter are as follows. Assuming noncoherent reception, we model the bit error probability of different PHY modes of the standard considering the imperfect energy capture caused by the multipath channel and the intra symbol interference. Fixing the transmit pulse energy, we also propose a novel LA scheme that can adapt the number of pulses per symbol to the channel conditions in order to maximize energy efficiency. In order to approximate the amount of captured energy in noncoherent IR-UWB receivers, two unbiased estimators are proposed.

\section{PRoblem Formulation}

Assume that a set of $N_{s}$ sensor nodes communicates with a master hub node. We consider the channel model CM3 [7] which models body-surface to body-surface channels, e.g. a sensor-sensor or sensor-hub, when the hub is in the vicinity of the sensors and is less than two cm away from the body. The hub uses management/control frames to adjust the PHY settings of the nodes.

We refer to each modulation and coding scheme (i.e. data rate) in the IEEE 802.15.6 standard as a physical layer mode for simplicity (see [1], Tables 67 and 68). The PHY modes are represented by an index $m, m \in[0, M-1]$ where $M$ is the number of available modes, and are referred to as "PHY $m$ ". We assume that the receiver can only select between PHY modes with the same modulation for simplicity. Hence, $M=6$ (PHY 0-PHY 5) in the default PHY, and $M=5$ (PHY 0PHY 4) in the high QoS PHY. Noncoherent reception based on energy detection (ED) with on-off signaling is assumed for the default PHY and autocorrelation (AC) reception with differential binary phase shift keying (DBPSK) is used for the high QoS PHY [1], [8].

Let $N_{p}$ denote the number of pulses per symbol and $p(t)$ be the pulse waveform of energy $E_{p}$ and duration $T_{p}$. The transmitted IR-UWB signals corresponding to the default and high QoS PHY can be expressed as

$$
\begin{aligned}
x_{D}(t) & =\sum_{i}\left[d_{i} w\left(t-c_{i} T_{w}-2 i \frac{T_{s}}{2}\right)+\right. \\
(1 & \left.\left.-d_{i}\right) w\left(t-c_{i} T_{w}-(2 i+1) \frac{T_{s}}{2}\right)\right], d_{i} \in\{0,1\}, \\
x_{H}(t) & =\sum_{i} d_{i} w\left(t-c_{i} T_{w}-i T_{s}\right), d_{i} \in\{+1,-1\},
\end{aligned}
$$

respectively, where $d_{i}$ is the $i$ 'th data symbol transmitted within the symbol time $T_{s}, w(t)=\sum_{k=0}^{N_{p}-1} p\left(t-k T_{p}\right)$ is a waveform of duration $T_{w}=N_{p} T_{p}$, and $c_{i}$ is the time hopping value in the range $0<c_{i}<N_{h}-1$, in which $N_{h}$ is the total number of hops. Regardless of the PHY option, there are 32 timeslots in each symbol duration. 
At the receiver, the amount of collected energy depends on the integration time $T_{i}$ as well as the channel's impulse response $h(t)$, and can be modeled by the energy capture index [9] defined as $\mu\left(T_{i} \mid h\right)=\int_{0}^{T_{i}}|h(t)|^{2} d t$, where $\mu\left(T_{i} \mid h\right) \in[0,1]$. Given $g(t)$ the received pulse waveform $(g(t)=p(t) * h(t)$, where $*$ denotes linear convolution), the instantaneous amount of captured energy of the received pulse is $\int_{0}^{T_{i}} g(t)^{2} d t=\mu\left(T_{i} \mid h\right) E_{p}$. We model $\mu\left(T_{i} \mid h\right)$ using the following exponential decay fit [8], [9] for CM3

$$
\mathbb{E}\left\{\mu\left(T_{i} \mid h\right\}=1-\exp \left[-\left(\frac{\left(T_{i}+15.56\right)}{46.22}\right)^{1.84}\right] .\right.
$$

The error performance of the noncoherent UWB systems has already been studied in [9] and [10]. However, since the standard uses a special terminology (e.g. on-off signaling that combines on-off keying with waveform coding), we briefly include the error analysis in this section to prevent confusion. We will also require the equations to estimate the energy capture index based on the decision statistic in Section III. Nevertheless, we complete the previous works on the error performance of noncoherent IR-UWB systems by incorporating the impact of intra symbol interference.

Let $T_{p}=2.003 \mathrm{~ns}$ (see [1]) and suppose the integration window covers a complete timeslot such that $T_{i}=T_{w}=$ $N_{p} T_{p}$. Therefore, intra symbol interference can occur in a timeslot when the channel's RMS delay spread is large enough compared with $T_{p}$. This is actually a constructive property. The overall captured energy per symbol can be increased since the multipath components of the earlier pulses can be captured during the integration time of the proceeding pulses.

At the receiver input, first a bandpass filter with bandwidth $B$ removes the unwanted spectra. The filtered signal is

$$
r(t)=\sum_{i} d_{i} \widetilde{w}\left(t-c_{i} T_{w}-i T_{s}\right)+\widetilde{n}_{i}(t),
$$

where $\widetilde{n}_{i}(t)$ is zero-mean band-limited Gaussian noise with two-sided power spectral density $\frac{N_{0}}{2}$ over the frequency band $B$ and the $i$ 'th symbol time, and $\widetilde{w}(t)$ and $\widetilde{g}(t)$ are the filtered version of $w(t)$ and $g(t)$ respectively.

\section{A. Default PHY}

From [10] and (5)-(7) ${ }^{1}$ on the next page, the conditional mean and variance of the decision variable in the default PHY, $Z_{D}$ are

$$
\begin{aligned}
& m_{Z_{D} \mid 1}=E_{p} \sum_{k=0}^{N_{p}-1} \mu\left(T_{w}-k T_{p} \mid h\right), \\
& \sigma_{Z_{D} \mid 1}^{2}=2 E_{p} N_{0} \sum_{k=0}^{N_{p}-1} \mu\left(T_{w}-k T_{p} \mid h\right)+2 N_{p} B T_{p} N_{0}^{2} .
\end{aligned}
$$

When the total number of samples $\left(2 N_{p} B T_{p}\right)$ is large, the sum pdf tends to the Gaussian distribution. Based on the justification in [10], this approximation is valid for $N_{p} B T_{p}>20$. This implies that when two $500 \mathrm{MHz}$ channels are used (as

\footnotetext{
${ }^{1}\left\{g_{j, k}\right\}$ and $\left\{n_{i, j, k}\right\}$ are independent samples of the band limited signals $\widetilde{g}(t)$ and $\widetilde{n}_{i}(t)$ within the specified time durations [10].
}

is mandatory by the 802.15 .6 standard), the approximation is valid for $T_{p}>2$ ns.

The bit error probability can then be approximated by

$$
P_{b} \approx Q\left(\frac{\bar{\mu}_{t} N_{p} E_{p} / N_{0}}{\sqrt{2 \bar{\mu}_{t} N_{p} E_{p} / N_{0}+2 N_{p} B T_{p}}}\right),
$$

where $Q(\cdot)$ is the tail probability of the standard normal distribution and $\bar{\mu}_{t}=\mathbb{E}\left\{\mu_{t}\right\}$ in which $\mu_{t}=$ $\sum_{k=0}^{N_{p}-1} \mu\left(T_{w}-k T_{p} \mid h\right) / N_{p}$.

\section{B. High QoS PHY}

From (11)-(13), the conditional mean and variance of the decision variable $Z_{H}$ corresponding to the high QoS PHY are

$$
\begin{aligned}
m_{Z_{H} \mid+1} & =\mu_{t} N_{p} E_{p}+N_{p} B T_{p} N_{0}, \\
\sigma_{Z_{H} \mid+1}^{2} & =\mu_{t} N_{p} E_{p} N_{0}+N_{p} B T_{p} N_{0}^{2} / 2 .
\end{aligned}
$$

The bit error probability of the DBPSK system with the Gaussian approximation is expressed as

$$
P_{b} \approx Q\left(\frac{\bar{\mu}_{t} N_{p} E_{p} / N_{0}}{\sqrt{\bar{\mu}_{t} N_{p} E_{p} / N_{0}+N_{p} B T_{p} / 2}}\right) .
$$

\section{THE PROPOSED SCHEME}

Having an expression for $P_{b}$, the calculation of packet success rate (PSR) and the energy efficiency of different PHY modes can be found in [11, equations (7) and (10)]. We can model the energy efficiency $\eta \in[0,1]$ of the PHY mode $m$ as

$$
\eta=\frac{\varepsilon_{1} L_{p}}{\varepsilon_{1}\left(L_{p}+L_{h}\left(R_{p} / R_{h}\right)\right)+E_{0}} \operatorname{PSR}\left(\gamma, \bar{\mu}_{t}, m\right)
$$

where $\gamma=E_{p} / N_{0}, \varepsilon_{1}$ represents the useful energy for communication of one bit of information, $L_{p}$ and $L_{h}$ are the length of data payload and overhead having rates $R_{p}$ and $R_{h}$ respectively, and $E_{0}$ is the total required extra energy which models the energy required for synchronization, data encoding/decoding, and transmission and reception of packet acknowledgment messages. For further details of the above model and typical values of the parameters see [11]. We assume $E_{p}$ is constant and is known at the hub.

Our LA is based on the $\left(\gamma, \bar{\mu}_{t}\right)$ pair. Assume a sensor $s$ $\left(s \in\left[1, N_{s}\right]\right)$ transmits data to the hub. The task of the LA algorithm is to identify for the hub the PHY mode $m_{s}^{*}$ a given sensor $s$ should select in order to maximize $\eta$ in (17).

$$
m_{s}^{*}=\underset{m}{\arg \max }\left\{\eta \mid\left(\gamma, \bar{\mu}_{t}, m\right)\right\}, m \in[0, M-1] .
$$

This mechanism is depicted in Fig. 1 for default and high QoS PHY. The solid lines represent full energy capture $\left(\bar{\mu}_{t}=\right.$ $1)$. Our simulations for imperfect energy capture are carried out assuming channel model CM3 and are demonstrated by dashed lines (the corresponding $\bar{\mu}_{t}$ values are listed in Table I at the end of this section). Note that the adaptation map in any channel condition is a version of the full-energy map that is randomly shifted to higher SNRs. Fig. 2 shows the adaptation maps for maximum energy efficiency. Obviously, the knowledge of $\bar{\mu}_{t}$ is required for selecting the optimal PHY mode. The benefit of using the pair $\left(\gamma, \bar{\mu}_{t}\right)$ instead of their 


$$
\begin{aligned}
& Z_{D}[i]=\int_{i T_{s}+c_{i} T_{w}}^{i T_{s}+\left(c_{i}+1\right) T_{w}}|r(t)|^{2} d t-\int_{(i+1 / 2) T_{s}+c_{i} T_{w}}^{(i+1 / 2) T_{s}+\left(c_{i}+1\right) T_{w}}|r(t)|^{2} d t \\
& \left.Z_{D}[i]\right|_{d_{i}=1}=\int_{0}^{T_{w}}\left[\sum_{k=0}^{N_{p}-1} \widetilde{g}\left(t-k T_{p}\right)+\widetilde{n}_{1}(t)\right]^{2} d t-\int_{0}^{T_{w}} \widetilde{n}_{1}^{2}(t) d t \\
& \left.Z_{D}[i]\right|_{d_{i}=1}=\sum_{k=0}^{N_{p}-1}\left(\sum_{j=0}^{2 B\left(T_{w}-k T_{p}\right)-1} g_{j, k}+\sum_{j=0}^{2 B T_{p}-1} n_{1, j, k}\right)^{2}-\sum_{k=0}^{N_{p}-1}\left(\sum_{j=0}^{2 B T_{p}-1}\left(n_{1, j, k}\right)^{2}\right) . \\
& Z_{H}[i]=\int_{i T_{s}+c_{i} T_{w}}^{i T_{s}+\left(c_{i}+1\right) T_{w}} r(t) r\left(t-T_{s}\right) d t, \\
& Z_{H}[i]=\int_{0}^{T_{w}}\left[\left(\sum_{k=0}^{N_{p}-1} d_{1} \widetilde{g}\left(t-k T_{p}\right)+\widetilde{n}_{1}(t)\right) \cdot\left(\sum_{k=0}^{N_{p}-1} d_{2} \widetilde{g}\left(t-k T_{p}\right)+\widetilde{n}_{2}(t)\right)\right] d t, \\
& \left.Z_{H}[i]\right|_{d_{1}, d_{2}=+1}=\sum_{k=0}^{N_{p}-1}\left[\sum_{j=0}^{2 B\left(T_{w}-k T_{p}\right)-1} g_{j, k}^{2}+\sum_{j=0}^{2 B T_{p}-1} g_{j, k} n_{1, j, k}+g_{j, k} n_{2, j, k}+n_{1, j, k} n_{2, j, k}\right] .
\end{aligned}
$$
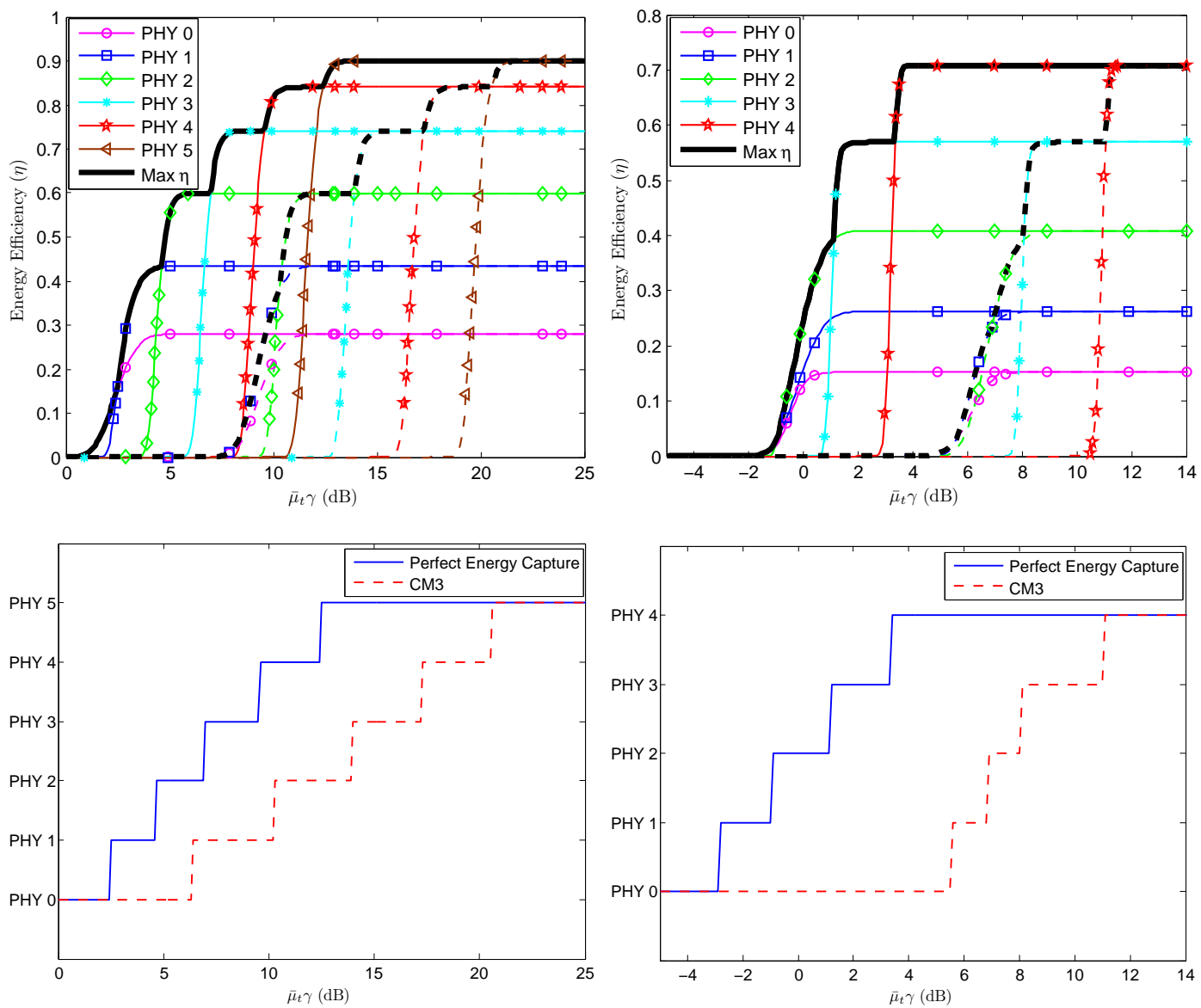

Fig. 1. Energy efficiency of different data rates and the optimal adaptation maps for the default (left) and high QoS (right) PHY, assuming full (solid) and imperfect (dashed) energy capture.

product, i.e. the effective SNR, is that the adaptation maps in Fig. 2 can be derived off-line and saved as a simple look-up table. Otherwise, (18) should be solved on every run of LA.

Considering (8) and (14), we can use the first moment of the decision variable to estimate $\bar{\mu}_{t}$. The following estimators over the interval $A=[0,1]$ can approximate the normalized energy capture index within the $i$ 'th symbol, based on $\tau$ recent samples of the decision variable corresponding to the 

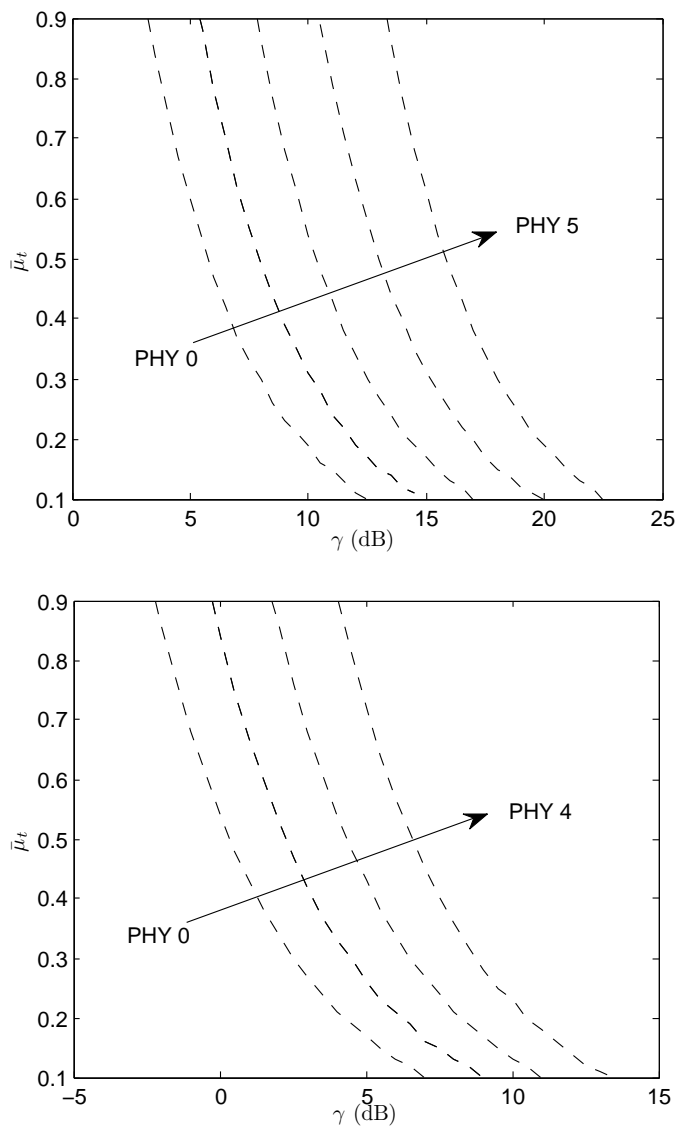

Fig. 2. The optimal adaptation maps corresponding to maximum energy efficiency default (top) and high QoS (bottom) PHY. The dashed lines represent the border between two neighboring PHY modes.

previously received symbols in the ED and AC receivers:

$$
\begin{aligned}
& \hat{\mu}_{D}[i]=\Psi_{A}\left(\frac{\sum_{j=0}^{\tau-1}\left|Z_{E D}[i-j]\right|}{\tau N_{p} E_{p}}\right), \\
& \hat{\mu}_{H}[i]=\Psi_{A}\left(\frac{\sum_{j=0}^{\tau-1}\left|Z_{A C}[i-j]-N_{p} B T_{p} N_{0}\right|}{\tau N_{p} E_{p}}\right),
\end{aligned}
$$

The hard-limiting function $\Psi_{A}(\cdot)$ is to make sure that the estimation is within the interval $A=[a, b]$, and is given by

$$
\Psi_{A}(x)=\left\{\begin{array}{lc}
b, & x \geq b \\
x, & a<x<b \\
a, & x \leq a .
\end{array}\right.
$$

After LA finds the optimal mode at the hub, it should send a management/control packet to the node to provide it with the optimal PHY mode.

The proposed scheme has been extensively simulated assuming different $\gamma$ values and the channel model CM3. The average values of $\bar{\mu}_{t}$ and $\eta$ are compared with the static system, i.e. fixed communication on different PHY modes in Table I (PHY 0 is the mandatory mode of the standard). Since the LA scheme uses all modes in different conditions, only the overall energy efficiencies are written for this scheme. The range of $\gamma$ (in $\mathrm{dB}$ ) is uniformly within $[-5,25]$ and $[4,14]$ for the default and high QoS PHY simulations, respectively. It can be seen that the proposed scheme can significantly improve the energy efficiency of the system. To quantify the estimation accuracy of the proposed estimators for the energy capture index, we also used (8), (9) and (14), (15) to generate random samples of the decision variable for a given pair $\left(T_{w}, \gamma\right)$, assuming $\tau=100$ and $E_{p}=1$ for simplicity. The achieved mean square error (MSE) for the estimators given in (19) and (20) is 0.0595 and 0.0523 , respectively.

TABLE I

SimULATION RESULTS

\begin{tabular}{lccccc} 
& $N_{p}$ & $T_{w}(\mathrm{~ns})$ & $\bar{\mu}_{t}$ & $\eta$ (Default) & $\eta$ (High QoS) \\
\hline LA & - & - & - & $\mathbf{0 . 5 8}$ & $\mathbf{0 . 4 3}$ \\
\hline PHY 0 & 32 & 64.103 & 0.62 & 0.22 & 0.12 \\
PHY 1 & 16 & 32.051 & 0.41 & 0.34 & 0.20 \\
PHY 2 & 8 & 16.026 & 0.27 & 0.44 & 0.29 \\
PHY 3 & 4 & 8.012 & 0.20 & 0.42 & 0.34 \\
PHY 4 & 2 & 4.006 & 0.17 & 0.35 & 0.22 \\
PHY 5 & 1 & 2.003 & 0.15 & 0.24 & -
\end{tabular}

\section{CONCLUSION}

We have proposed a novel link adaptation strategy for IEEE 802.15.6 IR-UWB systems that does not require channel estimation at the transmitter side. Assuming a constant pulse energy among different PHY modes, and considering different number of pulses recommended by the standard, the proposed adaptation scheme is based on the estimated SNR and the so called energy capture index. The energy capture index is estimated based on the recent samples of the decision statistic. Since it can provide a good measure for channel quality, the proposed scheme is sensitive to the temporal variations in channel conditions, noise, and interference. Therefore, it can correctly select the most energy efficient PHY mode to maintain the required levels of performance.

\section{REFERENCES}

[1] "IEEE standard for local and metropolitan area networks - part 15.6: Wireless body area networks," IEEE Std 802.15.6-2012, 2012.

[2] S. Fu, D. Wang, H. Zhai, and Y. Li, "An enhanced link adaptation for the mb-ofdm uwb system," in Wireless Communications and Networking Conference (WCNC), 2012, pp. 2070-2075.

[3] R. Al-Zubi and M. Krunz, "Interference management and rate adaptation in ofdm-based uwb networks," IEEE Transactions on Mobile Computing, vol. 9, no. 9, pp. 1267-1279, 2010.

[4] F. Martelli, R. Verdone, and C. Buratti, "Link adaptation in wireless body area networks," in Vehicular Technology Conference (VTC Spring), 2011.

[5] — , "Link adaptation in IEEE 802.15.4-based wireless body area networks," in International Symposium on Personal, Indoor and Mobile Radio Communications Workshops (PIMRC Workshops), 2010, pp. 117121.

[6] W.-B. Yang and K. Sayrafian-Pour, "Interference mitigation using adaptive schemes in body area networks," International Journal of Wireless Information Networks, vol. 19, no. 3, pp. 193-200, 2012.

[7] IEEE 802.15 WPAN task group 6 (TG6) body area networks. [Online] Available: http://www.ieee802.org/15/pub/TG6.html

[8] K. Witrisal, G. Leus, G. Janssen, M. Pausini, F. Troesch, T. Zasowski, and J. Romme, "Noncoherent ultra-wideband systems," IEEE Signal Processing Magazine, vol. 26, no. 4, pp. 48-66, 2009.

[9] S. Dubouloz, B. Denis, S. De Rivaz, and L. Ouvry, "Performance analysis of LDR UWB non-coherent receivers in multipath environments," in IEEE International Conference on Ultra-Wideband, 2005.

[10] P. Humblet and M. Azizoglu, "On the bit error rate of lightwave systems with optical amplifiers," Journal of Lightwave Technology, vol. 9, no. 11, pp. 1576-1582, 1991.

[11] M. S. Mohammadi, Q. Zhang, E. Dutkiewicz, and X. Huang, "Optimal frame length to maximize energy efficiency in IEEE 802.15.6 UWB body area networks," IEEE Wireless Communications Letters, 2014. 